\title{
La nazione del miracolo. L'Italia e gli italiani tra Storia, memoria e immaginario
}

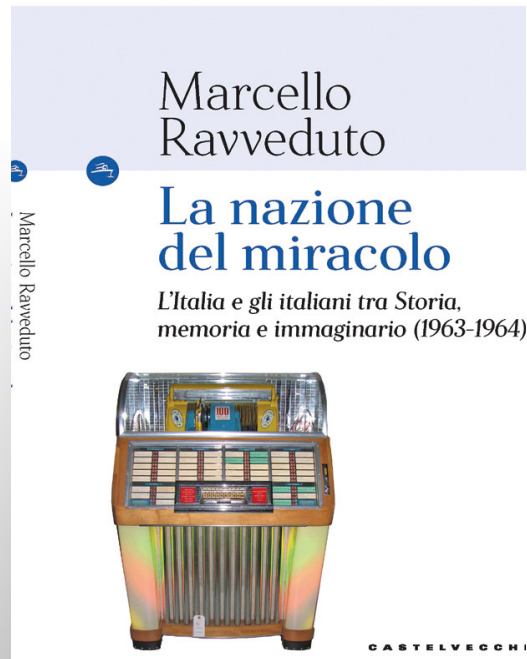

FICHA BIBLIOGRÁFICA

Marcello Ravveduto, La nazione del miracolo. L'Italia e gli italiani tra Storia, memoria e immaginario, Ediciones Castelvecchi, 2018, 192 páginas. ISBN: 978-88-3282-298-4.

\section{Patrizia Gabrielli I Università di Siena}

LA STORIA DELLE GRANDI TRASFORMAZIONI strutturali, sociali e del costume, che hanno investito l' Italia sul finire degli anni Cinquanta e i primi anni Sessanta, sono state al centro di diversi studi che hanno indagato il «boom» $\mathrm{o}$ «miracolo» da diversi punti di vista, a partire dalla stessa definizione, per molti impropria, del fenomeno. Sono state messe in luce innovazioni, persistenza di tradizioni, arretratezze, squilibri e contraddizioni, così come è stata ampiamente sottolineata la nascita di nuovi soggetti sociali. Dimostrando ampia padronanza della bibliografia disponibile, Marcello Ravveduto propone una monografia interessante e originale basata su fonti diversificate edite, è il caso delle memorie e delle testimonianze 
pubblicate in altri volumi su quella stagione, cui l'A. fa puntualmente riferimento, e inedite, come nel caso degli «home movies». Sebbene, stando al frontespizio, il biennio considerato è il 1963-64, che vede scemare la spinta propulsiva dell'economia italiana e lasciare il campo ad un rallentamento della "congiuntura», l'A. inquadra il passaggio nel più ampio arco cronologico a cavallo tra il Cinquanta e il Sessanta e, in sette capitoli propone una lettura che spazia dagli aspetti più prettamente economici a quelli culturali focalizzando l'attenzione sulla ridefinizione degli immaginari sociali. Questa, la definirei la sfida dell'A. e il tratto di maggiore originalità del suo più che apprezzabile lavoro. Una sfida che si fonda su una piena consapevolezza: «La realtà riflessa dai media, spesso in maniera alterata, compone l'immaginario che viene contenuto dalla memoria collettiva» (29), ed ancora «Il rapporto tra storia e immaginario non ci deve spaventare, rappresenta, piuttosto, un'occasione per conoscere gli elementi impliciti, istintivi ed emotivi che spesso sono assenti nella documentazione tradizionale» (30-31). Sebbene tante siano le storie di vita riportate da Marcello Ravveduto, l'asse centrale del racconto è data dalla vicenda di Ciro, nato nel 1936, parte di quella generazione che ha vissuto la tragedia della seconda guerra mondiale: "Ciro è il caso esemplare di una generazione che vive la difficile e lenta ricostruzione materiale e morale del paese. I giovani dell' immediato dopoguerra da una parte sperimentano un eccesso di esperienze, quali i traumi e le privazioni patite durante il conflitto, ma anche le aspirazioni derivanti dal nuovo regime democratico; dall'altra non hanno occasioni per esprimere l'insieme dei desideri contrastanti all'attenzione degli adulti» (60-61). Sebbene si rilevino sostanziali differenze tra le diverse regioni del Paese, sostanzialmente questa generazione vive «una condizione di debolezza sociale» a causa dalla bassa scolarizzazione, della precarietà, delle regole della famiglia patriarcale, tutti fattori che limitano l'autonomia decisionale (61). Vissuto in Campania, precisamente a Salerno, barbiere poi operaio sindacalizzato, Ciro è sposato con Anna, un'operaia. I coniugi sono genitori di tre bambini e, non trascurabile, padre e madre dell'A. che a un certo punto della narrazione rivela il legame con i protagonisti delle sue belle pagine. Una storia esemplare e paradigmatica quella di Ciro ed Anna, attraverso la quale, con grande partecipazione che non inficia l' interpretazione - a tale proposito merita richiamare l'attenzione sulle accurate pagine dedicate alla soggettività dello storico - Marcello Ravveduto restituisce al lettore un percorso di straordinaria emancipazione dalla precarietà, un cammino comune a tanti italiani, o meglio, la storia «di un' Italia contadina che si è sollevata sul piedistallo della modernizzazione» (75), la storia di milioni di donne e uomini che, non senza sacrifici e impegno, si sottraggono ad «una secolare povertà» (64).

Il dipanarsi di diverse storie individuali raccontate dagli stessi testimoni del boom, che l'A. trae in grande misura da altri volumi dedicati al tema, lascia emergere la metamorfosi della dimensione quotidiana, della dimensione materiale delle e degli italiani. Mutazioni dovute anche all'accesso ai consumi che produssero straordinari cambiamenti della vita materiale con consistenti miglioramenti delle condizioni di vita ed altrettanto rilevanti trasformazioni nella mentalità. Lavatrici, frigoriferi, ferri da stiro elettrici escono quotidianamente dalle piccole medio imprese che costellano il territorio delle regioni centro settentrionali. Questa diversa morfologia produttiva è tra le altre positive novità di quegli anni, che si traduce nel rafforzamento e in una caratterizzazione del mercato italiano e di interi settori capaci 
di assorbire manodopera garantendo un alto tasso occupazionale: processi, sottolinea a più riprese l'A., non indifferenti ai processi di costruzione della democrazia.

Gli elettrodomestici entrano nelle case degli italiani e sono accolti con grande soddisfazione dalle donne che vi intravvedono l'alleggerimento del lavoro domestico. La diffusione di automobili, specialmente delle utilitarie FIAT 600 e 500, interviene sui dati della produzione e sugli stili di vita mentre si realizzano, non senza limiti e speculazioni, tante opere volte a favorire la comunicazione, si pensi anche solo all'Autostrada del Sole la cui costruzione si è fissata nelle memorie di molti. Interventi che si riflettono con forza sulla mobilità e, insieme con il miglioramento del tenore di vita, introducono la diffusione delle vacanze estive e invernali, al mare, in montagna, sui laghi.

In questo quadro, Ravveduto assegna un ruolo particolare alla televisione. A partire dal 1954, anno delle prime trasmissioni Rai, e sempre più massicciamente dall'ultimo scorcio del decennio, la televisione si afferma quale oggetto amato e desiderato. Lo è anche per Ciro ed Anna: «Nel soggiorno, dove si consumano i pasti, la protagonista indiscussa è la televisione, posizionata di fronte al divano» (45). L'apparecchio contribuì, come è stato rilevato, alla diffusione della lingua nazionale, di una lingua tutt'altro che statica, che cambia «al cambiare delle condizioni», da qui l'introduzione di nuovi vocaboli ed espressioni coniate dai media (260). Importante anche il ruolo di alcune trasmissioni ai fini di una maggiore e diffusa conoscenza del patrimonio culturale italiano. Si pensi a proposito al ruolo degli sceneggiati di grande successo, al cinema, al varietà, allo sport, tutte manifestazioni che agevolano i processi di integrazione e di consolidamento del senso di appartenenza alla comunità. Se da un lato, nel lungo periodo la televisione indebolì la socializzazione familiare (come denunciava un'abbondante letteratura), dall'altra, alle origini, la sua limitata diffusione favorì nuove forme di sociabilità. Abbondano le testimonianze di coloro che per assistere alle trasmissioni più in voga, da Lascia o raddoppia? condotto dal popolarissimo Mike Bongiorno, a Canzonissima, piuttosto che il Festival della Canzone italiana, si radunavano nei bar, nelle osterie o nelle abitazioni di più fortunati vicini di casa. Da tali processi non può restare estraneo lo storico: «I media hanno stravolto il laboratorio dello storico in cui affluiscono, oltre ai tradizionali documenti cartacei, i suoni, le immagini e gli oggetti della cultura materiale. Fonti che trasudano memoria e che concorrono a "fare gli italiani" scandendone l'esistenza collettiva e avviandone una nazionalizzazione tumultuosa, gonfia di umori, di mode, di accensioni improvvise e tuttavia in grado di scardinare tutti i tradizionali riferimenti culturali ereditati dall'Ottocento» (19). I cambiamenti, rileva a ragione l'A., sono evidenti maggiormente tra i giovani, ormai diversi da Ciro ed Anna. Ragazzi e ragazze ascoltano una nuova musica che trova incarnazione negli «urlatori». Ampie tracce di questa mutazione generazionale, alla quale è dedicato in particolare il quinto capitolo, si rinvengono nella dimensione del consumo, che veicola oggetti e messaggi specifici, come nell'autorappresentazione, aspetto studiato sulla base di immagini e filmati. I giovani si differenziano dagli adulti anche in virtù di una rivoluzione estetica che sottolinea lo stacco generazionale con la conquista e la pratica di inediti spazi di libertà. Passaggi in tale direzione si colgono anche nella dimensione di genere. Le giovani donne con i loro atteggiamenti, abiti, colori, sembrano confermare che il cambiamento in atto le ha coinvolte: «vestono alla moda, indossano accessori vistosi, cominciano a truccarsi, bevono alcolici, fumano e non hanno remore a condividere momenti collettivi con 
l'altro sesso. La loro vita sta cambiando. Anche quando rimangono in casa hanno maggior tempo da dedicare a se stesse» (74).

Ampio spazio è dedicato alla dimensione politica e alle strategie di alcuni leader; le elaborazioni teorico politiche: su quest' ultimo aspetto merita menzione il paragrafo dedicato a "il Mulino". Sono ampie e argomentate le considerazioni su alleanze, programmi, riforme attuate e mancate. E' il caso della riforma Sullo sulla pianificazione urbanistica, al centro di contese ed aspre polemiche, attacchi personali: «Da allora chiunque abbia tentato di riordinare l'urbanistica è stato marchiato con la "sindrome di Sullo", una patologia infettiva che mette a rischio anche la più brillante carriera politica» (181). Interessanti i tanti richiami alle forme della propaganda, tra cui spiccano i documentari prodotti dalla DC e dal PCI a tale scopo che Ravveduto esamina sulla base delle specifiche competenze maturate nell'abito della Public and Digital History. Attraverso un'analisi critica delle posizioni espresse da partiti e alcuni intellettuali, l'A. sembra sollecitare il superamento di visioni e timori anacronistici rispetto alle grandi trasformazioni del tempo presente ed invita a tenere presente un dato: «l'accesso ai consumi nel secondo dopoguerra si è intrecciato con la definizione della cittadinanza repubblicana. I modelli di consumo svolgono una funzione di legittimazione sociali per le "classi subalterne", fino a quel momento escluse dal benessere, contribuendo alla stabilizzazione della democrazia» (101-102). 